cemoti $\begin{aligned} & \text { Cahiers d'études sur la Méditerranée } \\ & \text { orientale et le monde turco-iranien }\end{aligned}$

19 | 1995

Laïcité(s) en France et en Turquie

\title{
Ecole publique, Ecole privée et Laïcité en France
}

\section{Gustave PEISER}

\section{OpenEdition}

\section{Journals}

Édition électronique

URL : http://journals.openedition.org/cemoti/1699

DOI : 10.4000/cemoti.1699

ISSN : 1777-5396

Éditeur

AFEMOTI

Édition imprimée

Date de publication : 1 janvier 1995

ISSN : 0764-9878

\section{Référence électronique}

Gustave PEISER, «Ecole publique, Ecole privée et Laïcité en France », Cahiers d'études sur la Méditerranée orientale et le monde turco-iranien [En ligne], 19 | 1995, mis en ligne le 14 mai 2006, consulté le 07 septembre 2020. URL : http://journals.openedition.org/cemoti/1699 ; DOI : https:// doi.org/10.4000/cemoti.1699

Ce document a été généré automatiquement le 7 septembre 2020.

Tous droits réservés 


\title{
Ecole publique, Ecole privée et Laïcité en France
}

\author{
Gustave PEISER
}

La République est une philosophie avant d'être un régime ; elle est une Eglise, une Eglise laïque dont le dogme est la libre pensée et dont le prêtre est l'instituteur.

Ces fortes paroles d'Alain montrent à merveille le lien indissociable non seulement entre la laïcité et la République, mais aussi entre l'école laïque et la République. L'école laïque est aussi fortement ancrée dans le système républicain que l'est l'unité et l'indivisibilité de la République. Toutefois la conception de la laïcité que traduit cette formule pèche sur plusieurs points. Ainsi cette conception agressive de la laïcité, où l'on enseignerait un dogme de la libre pensée, correspond seulement à certaines tendances qui se sont faites jour, particulièrement entre 1880 et 1914, mais elle est loin de recouvrir toute la réalité. Surtout, et c'est là l'essentiel, Alain renverse d'une certaine manière l'ordre des facteurs : la République laïque serait née avant l'école laïque; l'école laïque aurait été créée pour perpétuer une République laïque déjà existante. Que l'école laïque constitue un outil, un instrument de consolidation du régime républicain, cela est indiscutable. Mais historiquement et cela est fréquent en France, l'ordre des facteurs est inversé : l'école laïque, ou de façon plus générale le caractère laïque du système d'enseignement est antérieur à la République laïque. A s'en tenir aux simples dates, la séparation des Eglises et de l'Etat, qui crée juridiquement la République laïque, ne date que de 1905. Les grands textes scolaires créant l'école laïque s'étalent de 1880 à 1886 . C'est bien en réalité sur le socle, sur les fondations de l'école laïque que se crée la République ; c'est la bataille pour l'école laïque et le triomphe de celle-ci qui permettra d'aboutir à la République laïque. Alors que les lois scolaires ou l'interdiction d'enseigner aux congrégations de 1901 sont encore considérées comme agressives, la loi de séparation des Eglises et de l'Etat est plutôt considérée comme une tentative de réconciliation.

En France, rien ne s'explique par la logique, tout par l'Histoire. Au lendemain de la défaite de 1871, le dépit national cherche d'abord un dérivatif dans l'exaltation religieuse développée par l'Eglise. Les laïques, l'école font peur. Mais les républicains qui vont peu à peu prendre le pouvoir, qui triompheront après le départ de Mac Mahon 
en 1877, vont rassurer la bourgeoisie par leur prudence en matière sociale et de droit de propriété. La bourgeoisie revient alors peu à peu à cet anticléricalisme qui est le fond de son tempérament. L'Église, elle, est antirépublicaine, et cette lutte contre la République passe par l'enseignement. Les nouveaux républicains estiment que l'Etat ne peut patronner un enseignement, centre d'hostilité au régime. On n'est pas en soi contre l'instruction religieuse, mais celle-ci postule l'entrée du prêtre ou du congrégationniste au sein de l'école officielle : on les excluera de l'école.

Le problème se pose de la même façon, mais selon des modalités un peu différentes, pour l'école privée. On verra que la liberté de création d'écoles privées, essentiellement catholiques, n'a jamais été réellement mise en cause, et elle sera même renforcée sous la Troisième République. Certes, il est vrai que les lois de 1901 et 1904, moment où le conflit pour la laïcité atteint son apogée, interdisent aux congrégations, autorisées ou non, tout enseignement public et même privé. La liberté de création et d'exercice de la liberté d'enseignement est ainsi atteinte. Mais après 1918, sans que les textes ne changent, les congrégations enseigneront avec l'accord tacite de l'Etat.

Le problème essentiel et qui hante notre histoire politique depuis plus d'un siècle, c'est celui des aides matérielles à l'enseignement privé. Débat insoluble sur le fait de savoir si les "fonds publics ne doivent aller qu'à l'école publique" ou si au contraire, l'instruction étant obligatoire, les parents étant libres d'envoyer leurs enfants à l'école chrétienne, il ne faut pas pénaliser les familles qui feraient ce choix et qui devraient payer deux fois : une fois comme tout contribuable, leurs impôts servant à la mise en oeuvre de l'école publique, une seconde fois en payant la scolarité de leurs enfants à l'école privée. Ce débat n'est pas clos et le rapport Vedel relatif à la modification de la loi Falloux de 1850 n'en constitue qu'un aspect.

On comprend dès lors que les républicains aient toujours été réticents, tout en admettant la réalité de la liberté de l'enseignement à considérer celle-ci comme une liberté républicaine. Contrairement aux autres grandes libertés (presse, réunion, association, etc.) la liberté de l'enseignement a été revendiquée par l'Eglise catholique antirépublicaine, et les débats à l'Assemblée constituante de 1945-1946 entre communistes et socialistes d'un côté, et MRP de l'autre, montrent à quel point cette lutte qui est plus de principe que réelle, est vive. Il faudra la loi Debré du 31 décembre 1959 et surtout la décision du Conseil constitutionnel de 1977 à propos de la loi Guermeur pour enfin reconnaître à la liberté de l'enseignement son statut de liberté républicaine.

On verra deux parties : la laïcité est le fondement de l'école publique; la laïcité pose le problème de l'existence de l'école privée.

\section{LA LAÏCITE, FONDEMENT DE L'ECOLE PUBLIQUE}

“École publique" est par définition laïque. C'est là son essence. Mais cette laïcité a revêtu différentes formes.

La première forme est celle d'une laïcité agressive, qui constitue elle-même une religion qui s'oppose aux religions chrétiennes. Elle s'affirme dès le début de la Troisième République. La seconde forme est celle d'une laïcité-neutralité. L'école se borne à ne pas choisir, elle laisse aux parents et aux élèves la liberté de choix : l'école est neutre. La troisième forme, plus récente, prend l'allure d'une laïcité "souple" qui peut prendre en compte des expressions politiques et religieuses diverses.

La laïcité-laïcisme 
Cette laïcité agressive qui s'affirme à partir des années 1880 constitue davantage une doctrine qu'elle ne reçoit d'applications institutionnelles réelles.

Dans sa lutte contre le confessionnalisme, les fondateurs de l'école publique ont senti la nécessité de donner une consistance mystique à la laïcité. A priori, dire que la laïcité consiste dans la tolérance de toutes les opinions, c'est une simple attitude. La laïcité ne serait alors qu'un simple comportement de tolérance, une attitude pragmatique. Certains ont alors senti la nécessité de rattacher la laïcité à un dogme. Sur quelles bases en effet, autres que la religion, fonder l'instruction morale ou civique? La "morale de nos pères" invoquée par Jules Ferry semble un peu insuffisante.

Peu à peu se dégage une doctrine déiste-rationaliste, sorte de kantisme à la française. S'y mêle la défense d'une République idéale. Le "solidarisme" de Léon Bourgeois va permettre de déboucher sur un idéalisme humaniste. L'observation scientifique dégage une loi de solidarité naturelle, universelle (biologique) et sociologique, l'interaction des individus, le groupement. Il y a là une loi (Kant) qui doit être respectée. Il s'agit d'une sorte de dette de reconnaissance, de tolérance fraternelle. Ferdinand Buisson parlera même de "foi laïque", foi sans credo, respect scrupuleux de la vérité, honorant les grands hommes tels que Pasteur et Hugo. Ce même culte sera poursuivi par Albert Bayet dans ses Leçons de morale de 1902. On sent aussi l'influence des écoles sociologiques de Lévy-Bruhl et de Durkheim. On met en avant le caractère relatif des religions. Les religions divisent, la science unit. C'est l'éthique du caractère universel de la recherche scientifique.

Sur le plan pratique, le laïcisme aboutira parfois en tant que dogme à combattre la religion. Selon Albert Bayet : "Nous poursuivrons la campagne d'émancipation laïque au cri de "à bas la neutralité"". Pour Clémenceau : "La guerre entre nous n'est pas dans les chemins creux, elle est dans l'école". Selon Viviani: "La neutralité de l'école fut toujours un mensonge; nous n'avons jamais eu d'autre dessein que de faire une université anti-religieuse, de façon active, militante, belliqueuse". Le même homme politique s'écrie en 1906 : "Nous nous sommes attachés à une oeuvre d'irréligion, nous avons arraché la conscience humaine à la croyance. Nous avons éteint dans le ciel des lumières qu'on ne rallumera pas...". Enfin Aulard écrit en 1903 : "Oui, nous voulons supprimer la religion pour cette raison que nous avons quelque chose de très supérieur à mettre à sa place : la philosophie laïque, fruit de la science et de la longue expérience de l'Humanité..."

Mais ce laïcisme peut éventuellement déboucher sur un nouveau dogmatisme. Le dogmatisme politique peut ou pourrait utiliser l'école; il en va de même des philosophies. Ainsi pour les marxistes, les manifestations religieuses sont un aspect de la superstructure des sociétés. Les rivalités confessionnelles constituent des modalités de la lutte des classes. Dans un contexte différent, les aliments du conflit disparaîtraient. Il n'y aura plus de classes possédantes obligées d'entretenir des représentations religieuses pour entretenir leur domination. Ainsi l'école laïque ne serait qu'un moment de l'évolution historique, la volonté de la bourgeoisie de contrôler l'école. Ce moment sera dépassé, l'école laïque n'est qu'une transition, non une fin. Les marxistes rejetteront la neutralité intégrale. Pour Pivert "la neutralité apparente entre les philosophies qui flotte sur les classes et les époques est impraticable" (Eglise et Etat). Il est bien évident que cette conception d'un laïcisme marxisant ne fut jamais une politique officielle; elle resta l'expression politique de quelques auteurs ou de quelques enseignants. 
Si l'on revient au laïcisme plus traditionnel de lutte contre l'Eglise, il ne trouve d'ailleurs lui non plus guère de reflet dans l'aménagement pratique de l'école. Les créateurs de l'école publique, laïques convaincus, sont souvent, sur un plan plus général, des libéraux. Peut-être trouverait-on une application pratique de ce laïcisme agressif - mais encore est-ce affaire d'opinion - dans le fait qu'à l'école primaire aucune instruction religieuse ne saurait être donnée au sein des bâtiments scolaires. Laïcisme agressif peut-être aussi lorsque le ministère de l'Instruction publique refuse après avis du Conseil supérieur de l'instruction publique d'interdire des livres portant atteinte au principe de neutralité : mais le Conseil d'Etat a toujours sanctionné ces refus (8 avril 1911, Rosselin).

La laïcité-neutralité

C'est cette idée de neutralité qui domine en réalité le système français. Idéologiquement, la neutralité s'est peu à peu imposée ; dans les faits et les institutions, elle a toujours existé.

La laïcité-neutralité consiste d'une part à bouter l'Eglise hors de l'école, mais aussi, et cela est plus spécifiquement français, le caractère neutre, non-confessionnel de tout l'enseignement. Gambetta affirme dès les débuts de la République : "Je désire de toute la puissance de mon âme, non seulement qu'on sépare les Eglises de l'Etat, mais qu'on sépare les écoles de l'Eglise. C'est pour moi une nécessité d'ordre politique et j'ajouterai, d'ordre social".

En réalité, l'école publique existait déjà avant 1870. Dans la mesure où la loi Guizot de 1830 et la loi Falloux de 1850 avaient permis l'ouverture d'établissements privés, la prédominance de l'Eglise avait commencé à disparaitre de l'école publique. De plus la liberté de l'enseignement supérieur sera mise en oeuvre par la République elle-même, en 1875.

Mais il s'agit maintenant d'aller plus loin, d'éliminer la confession de l'école. Cette particularité française est liée à l'Histoire nationale. La République l'emporte en 1878. Il s'agissait pour les républicains d'étendre par le moyen de l'obligation scolaire, de la gratuité scolaire, l'instruction populaire. Or, le rôle que l'Eglise catholique attribue à l'enseignement dans la lutte antirépublicaine, souvenir des luttes constantes entre clergé et administration universitaire, va rallier les républicains à des principes nouveaux. L'instruction religieuse postule l'entrée du prêtre ou du congrégationniste au sein de l'école publique. Cela créerait un climat de tension et faciliterait la propagande antirépublicaine. Le principe de Crémieux l'emporte : "Le prêtre à l'église, l'instituteur à l'école"...

C'est l'obligation scolaire, réunissant des enfants de toutes confessions, dans une même école, qui impose la laïcité. Le raisonnement est logique, la liaison n'est que partielle. L'application du principe aurait dû se faire dès 1833 ou 1850. La laïcité de 1882 est une conséquence politique; l'Eglise se bat à cette époque contre le principe de l'obligation scolaire, l'attentatoire aux droits des familles et moyen de coercité pour imposer un enseignement d'Etat" ; elle bataille contre la gratuité "car il n'y a de gratuité que ce qui l'est pour tous, ou l'école publique n'est pas l'école de tous", l'Eglise lutte aussi contre les internats de jeunes filles.

Si l'Eglise est doublement hors de l'école, celle-ci doit être neutre. Il existe une longue et célèbre lettre de Jules Ferry qu'il adresse aux instituteurs et dont nous citerons cet extrait : - "Dans les textes, il y a deux dispositions qui se complètent sans se contredire; d'une part, elle met en dehors du programme obligatoire l'enseignement de tout dogme 
particulier; d'autre part, elle y place au premier rang, l'enseignement moral et civique. Enseignement moral, oui, mais neutre. Si parfois vous étiez embarrassé pour savoir jusqu'où il vous est permis d'aller dans votre enseignement moral, voici une règle pratique. Au moment de proposer aux élèves un précepte, une maxime quelconque, demandez-vous vous s'il se trouve à votre connaissance un seul honnête homme qui puisse être froissé de ce que vous allez dire. Demandez-vous si un père de famille, je dis un seul, présent à votre classe et vous écoutant, pourrait de bonne foi refuser son assentiment à ce qu'il vous entendrait dire. Si oui, abstenez-vous de la dire, sinon ditesle hardiment. Vous ne toucherez jamais avec trop de scrupule à cette chose délicate et sacrée qu'est la conscience de l'enfant. »

Cette neutralité, c'est le choix de laisser le choix. Elle ne s'oppose pas à la religion, seulement elle n'y participe pas.

Cette laïcité-neutralité se ressent très fortement dans tout le dispositif mis en vigueur par les lois scolaires. La neutralité est plus forte dans le primaire que dans le secondaire, dans le secondaire que dans le supérieur.

Dans l'enseignement primaire il n'y aura pas de croix dans les bâtiments; aucune instruction religieuse ne pourra y être donnée. Le personnel est laïque. La neutralité implique une stricte impartialité à l'égard des religions. Les juges la font respecter : le Tribunal des conflits sanctionne un instituteur pour avoir tenu dans sa classe devant ses élèves des propos injurieux pour la religion (TC, 2 juin 1908, Morisot), le Conseil d'Etat accueille le recours de parents d'élèves contre le refus du ministère de l'Intérieur d'interdire des manuels portant atteinte à la neutralité. Mais la liberté de conscience des instituteurs est sauvegardée s'ils manifestent une stricte réserve.

Même dans l'enseignement primaire, l'instruction religieuse est favorisée. Les écoles vaqueront un jour par semaine en dehors du dimanche pour permettre, si les parents le désirent, de faire poursuivre une instruction religieuse à leurs enfants. Il ne peut $\mathrm{y}$ avoir enseignement religieux pendant les heures de classe. Ce problème du mercredi ou du jeudi libre soulève depuis peu de nouvelles difficultés, les parents voulant souvent faire remplacer le milieu de semaine par la liberté du samedi pour permettre aux enfants de partir avec les parents. Cette conception se heurte à de fortes réserves de l'Eglise.

Dans l'enseignement secondaire, il n'y a pas de croix dans les bâtiments, il n'y a pas de cours de religion assurés par le personnel enseignant. En ce qui concerne le personnel, il n'y a pas de loi interdisant aux prêtres d'enseigner, et on a juste le vieil arrêt Abbé Bouteyre de 1912, interdisant aux prêtres de se présenter au concours d'agrégation de philosophie. Mais cette jurisprudence est-elle encore valable? Surtout, une instruction religieuse peut être donnée dans les bâtiments scolaires, en dehors des heures de classe, par des aumôniers, pour lesquels la loi de 1905 prévoit que les dépenses pourront être inscrites aux budgets des collectivités publiques.

Ces principes ont été repris par la loi du 31 décembre 1959: "L'Etat prend toutes dispositions utiles pour assurer aux élèves de l'enseignement public, la liberté des cultes et de l'instruction religieuse...". Le décret du 22 avril 1960 fixe les modalités d'application de ce principe: dans les établissements comportant un internat, l'institution d'une aumônerie pour les différentes cultures est de droit lorsqu'elle est demandée par des parents d'élèves et l'enseignement religieux peut être donné dans l'établissement. Pour ceux qui ne comportent pas d'internat, la décision, sur demande des parents, appartient au recteur. 
La laïcité ouverte

Le problème de la laïcité s'est posé avec une particulière acuité dans l'affaire des "foulards islamiques". Mais la question posée est maintenant tout à fait différente : il ne s'agit plus de se battre contre la prééminence d'une religion. Maintenant, le problème posé est celui de la manifestation d'opinions religieuses ou non par les usagers des services publics. Une interprétation stricte du principe de neutralité obligerait les usagers à ne pas manifester leurs opinions de quelque façon que ce soit. Cette idée a prévalu longtemps étant donné la vivacité du combat de l'Eglise et de l'Etat puis, dans les années 1930-1940, la vivacité des combats politiques. Le Conseil d'Etat considère comme une mesure d'ordre intérieur, l'interdiction faite en 1935-36 par un proviseur de lycée, de porter tout insigne politique, même les couleurs nationales. Mais à partir de 1968, les mentalités ont évolué et des interprétations plus souples étaient possibles. La neutralité du service peut être respectée sans que des dispositions spécifiques trop contraignantes ne pèsent sur les usagers. La laïcité permettrait aux opinions de s'exprimer. On rejoint alors les textes internationaux contemporains tels que la Convention européenne des droits de l'homme (art.9) ou le Pacte de 1966 relatif aux droits civils et politiques. Ces textes prévoient la "liberté de manifester sa religion ou sa conviction, individuellement ou collectivement, en public ou en privé".

Il y a donc des modifications. La Cour européenne des droits de l'homme a reconnu applicable l'article 9 en ce qui concerne les services publics. La loi française du 10 juillet 1989 a également reconnu le droit d'expression des élèves dans les établissements d'enseignement public. On ne fait pas allusion toutefois à la liberté religieuse. Mais le Conseil d'Etat dans son avis rendu public relatif au foulard islamique, la circulaire Jospin, ainsi que le Conseil d'Etat dans sa décision Kherouaa (CE.2 nov. 1992) ont fait prévaloir une interprétation souple. Ils mettent en avant le "respect de la liberté des élèves" ainsi que le fait que la "liberté ainsi reconnue comporte pour eux le droit d'exprimer et de manifester leurs croyances religieuses à l'intérieur de l'établissement scolaire".

Cette vision des choses comporte des conséquences complexes et multiples. Ainsi le juge refuse toute interdiction générale et absolue. Le Conseil d'Etat a ainsi annulé les dispositions $\mathrm{du}$ proviseur du collège prohibant "le port de tout signe distinctif, vestimentaire ou tout autre d'ordre religieux, politique ou philosophique". La liberté est encadrée. Elle n'est pas absolue et s'arrête lorsqu'elle porte atteinte à la liberté d'autrui.

En premier lieu, le port d'un signe extérieur d'appartenance à une religion ne peut porter atteinte aux activités d'enseignement, au contenu des programmes et à l'obligation d'assiduité. L'assistance est ainsi obligatoire aux activités sportives. Le port d'un insigne religieux peut être interdit s'il gêne l'enseignement (travaux pratiques, activités sportives etc.).

En second lieu, le port de l'insigne ne doit pas constituer un acte de prosélytisme. Dans l'affaire Rudent de 1985 le juge a estimé que le principe de neutralité est incompatible avec des réunions publiques. Il en irait de même des réunions religieuses.

En troisième lieu, la protection de l'élève est prise en considération, par exemple s'il arbore des signes portant atteinte à sa dignité ou lorsqu'il y a menace pour sa sécurité ou sa santé (par exemple, obligation légale de vaccination, même si on y est opposé).

Enfin il y a le trouble de l'ordre public. Peut-on interdire le port du foulard qui manifeste la différence entre l'homme et la femme et qui constitue ainsi atteinte au 
principe d'égalité, principe fondamental de la République ? Ce rôle donné à la femme par le port du foulard pourrait éventuellement porter atteinte aux valeurs de la République. C'est pour cette raison que ce vêtement a entraîné un débat conflictuel. Pour le moment, le juge a refusé de donner une signification au port du foulard, mais le débat n'est pas clos.

\section{LA LAÏCITE ET LE PROBLEME DE L'EXISTENCE DE L'ECOLE PRIVEE}

La laïcité de la République et de l'école publique pose le problème de l'existence d'une école privée non laïque, confessionnelle, concurrente de l'école publique laïque. Il faut toutefois s'entendre sur la signification du terme "existence". Malgré les apparences, la question de la "création" d'écoles privées, de la libre ouverture d'écoles privées n'a guère fait l'objet de discussions. Certes, sous le régime du monopole napoléonien, la question a pu se poser, mais les régimes démocratiques, et en particulier la République laïque, n'ont pas mis en cause l'ouverture pratiquement sans limites d'écoles privées. Sous la Monarchie de Juillet, une loi de 1833 prévoit la possibilité de création d'écoles primaires libres; la loi Falloux de 1850 remplit le même office pour l'enseignement secondaire. La Troisième République reconnaît la liberté de l'enseignement supérieur en 1875. Si la loi prescrit quelques modalités de contrôle par la puissance publique relatives à la moralité, à la qualité des enseignants, au programme enseigné ou à la salubrité des locaux, il ne s'agit là que de règles tout à fait traditionnelles et normales et auxquelles toutes les écoles sont soumises dans des pays similaires à la France.

Les problèmes sont ailleurs. La principale question, insoluble semble-t-il, est celle de l'existence matérielle des écoles privées. La seconde question est celle du caractère "propre" de ces établissements.

L'existence matérielle des écoles privées

Les positions sont traditionnellement tranchées. A l'affirmation "fonds publics à école publique" s'oppose celle de la participation des écoles privées au service public de l'enseignement; les écoles privées doivent donc être subventionnées afin que les parents qui font ce choix pour leurs enfants n'aient pas à payer deux fois.

L'histoire est ancienne. Les écoles secondaires et techniques ont pu recevoir des subventions dans le cadre limité de la Loi Falloux de 1850. Mais en ce qui concerne les écoles primaires, l'interdiction des subventions était complète et la jurisprudence du Conseil d'Etat très ferme. Si on met de côté le régime de Vichy, des modifications vont apparaître sous la Quatrième République. En 1948, un décret Poinsot-Chapuis permet de donner des aides à toutes les familles nécessiteuses; la loi Marie de 1951 étend certaines bourses aux élèves de l'enseignement privé. La loi Barangé du 28 septembre 1951 permet de verser de l'argent aux associations de parents d'élèves.

C'est la loi Debré du 31 décembre 1959, adoptée après maintes crises, démissions et manifestations, qui constitue la véritable charte de l'aide à l'enseignement privé. L'aide est accordée non à l'enseignement privé, mais à certains établissements d'enseignement privé. Ceux-ci ont d'abord le choix du statu quo ou de leur intégration dans l'enseignement public. Mais deux autres voies leur sont ouvertes: le "contrat simple" et le "contrat d'association".

- Le "contrat simple" qui en principe ne devait être que temporaire, sera pérennisée en 1971, mais pour les seuls établissements primaires. Les établissements ouverts depuis cinq ans au moins et réunissant un certain nombre de conditions, peuvent passer des contrats pour toutes les classes ou seulement certaines d'entre elles. En contrepartie, ils doivent préparer aux examens officiels. Les maîtres qui ne sont pas 
nommés par l'Etat, reçoivent leur traitement directement de l'Etat qui ne paie pas les charges sociales. Aucune charge spécifique n'est imposée aux départements et aux communes.

- Le "contrat d'association" est ouvert à tous les établissements d'enseignement privé s'ils "répondent à un besoin scolaire reconnu". Les enseignements doivent être dispensés selon les règles générales de l'enseignement public. Surtout les maîtres, qui doivent avoir des titres équivalents à ceux du public, sont liés à l'Etat par contrat. La totalité de leur rémunération est versée par l'Etat, y compris les charges sociales. De plus, l'Etat, les départements, les communes doivent prendre en charge une partie des dépenses de fonctionnement.

Ces contrats ont eu un succès foudroyant et mettent fin au caractère "à part" de l'enseignement privé qui est désormais censé participer à la mission de service public de l'enseignement. C'est une véritable révolution qui entraînera modifications et soubresauts dans les années postérieures.

Une loi Guermeur de 1977 amplifie nettement l'aide à l'enseignement privé. En revanche, la gauche au pouvoir tente entre 1981 et 1984, par le moyen du projet Savary, de créer un grand service public de l'enseignement, tout en ne supprimant pas, contrairement à ce qui a été affirmé, l'enseignement privé. Le projet de loi Savary prévoyait que pour le privé l'Etat ne paierait plus à "guichet ouvert", pratiquement sans contrôle des besoins réels; il prévoyait une participation des responsables des collectivités locales aux délibérations budgétaires des lycées et collèges privés ainsi que la création d'établissements d'intérêt public entre l'Etat, les collectivités, les groupements et personnes de droit privé. Surtout, et ce fut une des raisons essentielles de l'échec du projet, il prévoyait la possibilité pour les maitres liés à l'Etat par contrat d'être titularisés comme fonctionnaires, s'ils le désiraient, après six ans. Quoi qu'il en soit, le projet souleva une tempête de protestations, des réunions publiques dont une de près d'un million de personnes à Paris. Le ministre de l'Education, Alain Savary, démissionna, le projet fut abandonné. La loi Chevènement du 28 janvier 1985 calma les passions, abandonnant les avantages trop extravagants de la loi Guermeur pour revenir à la loi Debré de 1959. La paix scolaire sembla s'installer, mais le feu continua à couver sous la cendre.

En 1991, le Conseil d'Etat censura les décisions de conseils généraux qui avaient pris à leur charge les dépenses d'investissement de collèges privés au-delà de la limite de $10 \%$ autorisés par la loi Falloux de 1850. La lutte se déplace à partir de ce moment vers l'abrogation de la loi Falloux considérée en 1993 comme défavorable à l'école privée, alors qu'en 1850 elle avait justement satisfait les voeux de celle-ci. Mais les temps changent. La loi Falloux a donc été abrogée fin 1993 pour permettre aux collectivités locales d'aider les établissements d'enseignement privé par des dépenses d'investissement plus substantielles. On sait que le Conseil constitutionnel a déclaré cette loi inconstitutionnelle, ce qui rétablissait la loi Falloux en l'état.

Le débat n'est pas simple. Abandonner les subventions à l'école privée qui participe effectivement à la mission d'enseignement est aujourd'hui impossible. Mais traiter les écoles privées de la même manière que l'école publique, c'est la fin du symbole de l'école de la République, école pour tous, et c'est l'un des fondements de la République laïque qui s'écroule. L'école privée a droit à l'existence, certes, mais elle n'est pas l'école de tous.

Le caractère propre de l'école privée 
La loi du 31 décembre 1959 affirme bien le caractère propre des établissements qui passent des contrats, le respect de leur spécificité. On comprend aisément que l'Eglise ait exigé le maintien de ce "caractère propre", mais l'école privée n'est pas alors l'école de tous les enfants.

Il faut reconnaître que les difficultés sont nombreuses et qu'il y a quelque difficulté à les objectiviser. Lorsque les établissements d'enseignement privé passent des contrats, ils s'obligent par là à accepter tous les élèves quelle que soit leur origine. Mais ce sont tout de même surtout des "élèves catholiques" qui vont dans l'enseignement privé, même si des élèves d'autres confessions ou sans confession s'y retrouvent aussi. Il est vrai également que l'Etat exerce un contrôle pédagogique afin que l'enseignement soit donné conformément à la loi du 31 décembre 1959, dans le respect absolu, selon les textes, de la liberté de conscience. Mais il n'empêche que le projet pédagogique global est bien inspiré par une religion, il y a des cours de religion; les enseignants peuvent être des prêtres, même si ceux-ci deviennent très nettement minoritaires. Tout ceci est d'ailleurs normal et peu critiquable. Mais comment concilier cela avec l'idée de la laïcité de la République "qui ne subventionne aucun culte" ?

Ce caractère spécifique, propre, des établissements privés catholiques, reste en fait très confus. Plusieurs décisions de jurisprudence y font allusion. Dans une importante affaire Dame Roy contre Association pour l'Education populaire Sainte-Marthe, il s'agissait du licenciement par un établissement privé d'une enseignante divorcée qui s'était remariée. Après trois arrêts de Cour d'appel et trois arrêts de la Cour de cassation, il est finalement admis (en 1978) qu'on devait retenir le caractère "propre" de l'établissement, et que celui-ci n'avait commis aucune faute de nature à conférer au licenciement un caractère abusif. Cet arrêt définitif fut tout de même critiqué. En revanche, le Tribunal administratif de Versailles dans une décision de 1987 annule un article d'un règlement interne d'un établissement privé sous contrat d'association, article relatif à l'obligation de "réserve". Cet établissement a pour caractère propre d'être un établissement d'enseignement catholique; il veut vivre les valeurs évangéliques suivant son projet éducatif. Tout personnel, qu'il soit salarié ou non de l'établissement, s'engage à respecter ce caractère propre. L'attitude de l'établissement n'est donc pas contraire à la liberté de conscience mais l'article en question ne peut figurer au sens du Code du Travail dans le règlement intérieur.

Conclusion

Laïcité, école publique, école privée. La laïcité du système scolaire se trouve confrontée à un double problème :

Comment respecter à l'intérieur de l'école publique le principe de la laïcité lorsqu'une sorte de "multiconfessionalité" semble prendre le dessus. Pour certains, une tolérance multiconfessionnelle à l'intérieur de l'école, sur le mode d'autres pays démocratiques devrait supplanter le système de l'école neutre de "la religion hors de l'école". A notre point de vue, ce nouveau principe multiconfessionnel serait fondamentalement contraire à ce qui a fait et fait encore la grandeur de l'école républicaine: son extraordinaire puissance d'intégration dans la société française. Aucun autre système, aussi tolérant soit-il, ne pourra le remplacer et les exemples a contrario de l'étranger le prouvent. Que l'école publique soit plus ouverte aux multiplicités culturelles est une chose, le maintien strict de la laïcité en est une autre.

Quant à l'école privée, si elle doit être aidée, subventionnée, elle ne peut devenir l'égale de l'école publique. Seule l'école publique est l'école de tous, celle de la République 
française ; elle est la racine du pays. Cette école publique ne peut devenir, comme l'ont préconisé certains récemment, une autre école qui aurait son caractère "propre" et spécifique, l'école de la liberté et de l'égalité. L'école publique est bien plus que cela, elle est la base du système démocratique français. 